\title{
Genomic relationships of the North American isolate of infectious salmon anemia virus (ISAV) to the Norwegian strain of ISAV
}

\author{
S. Blake ${ }^{1}$, D. Bouchard ${ }^{2}$, W. Keleher ${ }^{2}$, M. Opitz ${ }^{3}$, B. L. Nicholson ${ }^{1, *}$ \\ ${ }^{2}$ Department of Biochemistry, Microbiology and Molecular Biology, University of Maine, Orono, Maine 04469, USA \\ ${ }^{2}$ MicroTechnologies, Inc., Richmond, Maine 04357, USA \\ ${ }^{3}$ Animal Disease Diagnostic Laboratory, University of Maine, Orono, Maine 04469, USA
}

\begin{abstract}
Nucleotide and deduced amino acid sequences were determined for a 436 bp reverse transcriptase-polymerase chain reaction (RT-PCR) cDNA fragment from genome segment 8 and a 1151 bp RT-PCR cDNA fragment from genome segment 2 of the North American isolate of infectious salmon anemia virus (ISAV) and compared to the published sequences of Norwegian isolates of ISAV. The North American ISAV isolate exhibited $82.9 \%$ identity with the Sotra 92/93 ISAV isolate from Norway in the partial cDNA sequence of genome segment 2 , which encodes a polymerase component protein (PB1). The North American ISAV exhibited 88 and $89 \%$ identity with 2 partial cDNA sequences of genome segment 8 (nonstructural, NS, gene) reported for the Glesvaer/2/90 isolate from Norway. The North American ISAV exhibited $96.6 \%$ similarity with the Sotra $92 / 93$ ISAV isolate from Norway in the deduced amino acid sequences of the PB1 protein. The deduced amino acid sequence of the protein encoded in the partial cDNA fragment of open reading frame (ORF) 1 of genome segment 8 of the North American ISAV exhibited only 71.2 and $66.7 \%$ similarity with the 2 sequences of the Norwegian Glesvaer/2/90 isolate. However, the North American ISAV isolate exhibited 96.2 and $87.2 \%$ similarity with the 2 sequences of the Norwegian Glesvaer/2/90 isolate in the deduced amino acid sequences of the protein encoded in the partial CDNA of ORF 2. Comparison of these partial cDNA nucleotide and deduced amino acid sequences confirmed that the North American isolate is ISAV. However, the differences observed in these genomic sequences suggest that the North American isolate may represent a distinct genomic variant from the previously described Norwegian strains.
\end{abstract}

KEY WORDS: Infectious salmon anemia virus - Nucleotide sequence

Infectious salmon anemia virus (ISAV) is the etiological agent of a serious disease in Atlantic salmon Salmo salar L. which has been observed in Norway for the last 15 yr. ISAV virus has been shown to be an enveloped

- Addressee for correspondence.

E-mail: brucen@maine.maine.edu virus, 100 to $130 \mathrm{~nm}$ in diameter, which replicates by budding from the membrane of infected cells (Hovland et al. 1994, Dannevig et al. 1995, Nylund et al. 1995). Recently the genome of ISAV has been characterized by Mjaaland et al. (1997). The genome consists of 8 segments ranging in size from 1.0 to $2.3 \mathrm{~kb}$, with a total molecular size of approximately $14.5 \mathrm{~kb}$. Based on these morphological and replication data, these investigators concluded that ISAV resembles members of the viral family Orthomyxoviridae. The nucleotide sequence has been reported for a partial cDNA clone of the second largest genome segment (segment 2) of ISAV isolate Sotra 92/93 from Norway (Krossoey et al. unpubl., GenBank accession no. AJ002475). Genome segment 2 encodes the polymerase component protein PB1 of orthomyxoviruses. Two partially overlapping cDNA sequences have been reported for the smallest genome segment (segment 8) for the Glesvaer/2/90 ISAV isolate from Norway (Devold et al. unpubl., GenBank accession no. AJ012285; Mjaaland et al. 1997. GenBank accession no. Y10404). Segment 8 encodes 2 nonstructural (NS) proteins. No significant homology was found between the sequences of these Norwegian ISAV isolates and other orthomyxoviruses.

Recently, a new disease, originally called hemorrhagic kidney syndrome (HKS), has appeared in Atlantic salmon cultured in the Bay of Fundy, New Brunswick, Canada. HKS is associated with high levels of mortality in affected fish farms. Although not identical, some of the clinical signs characteristic of HKS were similar to those previously described for ISA. As described by Bouchard et al. (1999-this issue), we isolated a virus from moribund Atlantic salmon exhibiting HKS which we identified as ISAV on the basis of morphology and a reverse-transcriptase polymerase chain reaction (RT-PCR) assay using ISAVspecific primers. 
Table 1. Primers used in RT-PCR of North American ISAV isolate

\begin{tabular}{|lcl|}
\hline Primers & Polymerase gene (PB1) & Partial cDNA RT-PCR target \\
\hline Upstream & NS gene \\
Downstream & 5'-CTGCTGACTGGGGAAGATTC-3' & 5'-GGCTATCTACCATGAACGAATC-3' ' \\
'Previously described by Mjaaland et al. (1997) & 5'-GCGAAGTGTAAGTAGCACTCC-3' \\
\hline
\end{tabular}

In this investigation, we compared the genomic relationship of this North American isolate of ISAV with the Norwegian strains of ISAV using partial CDNA sequences of the NS proteins encoded in genome segment 8 and the PB1 encoded by genome segment 2 .

Materials and methods. Virus: The North American strain of ISAV was propagated in the chinook salmon embryo (CHSE-214) cell line (Lannan et al. 1984) at $15^{\circ} \mathrm{C}$ as described by Bouchard et al. (1999).

$\boldsymbol{R}$ T-PCR: RNA extraction and reverse transcription were performed as described previously (Blake et al. 1995). ISAV-specific primer sets (Table 1) were developed using the Oligo Primer software program (National Biosciences, Inc. [NBI], Plymouth, MN, USA) based on published sequences of the Norwegian ISAV strain (Mjaaland et al. 1997, GenBank accession no. Y10404, Krossoey et al. unpubl., GenBank accession no. AJ002475). These primers were used to amplify a 493 bp cDNA fragment from genome segment 8 and a $1209 \mathrm{bp}$ fragment from genome segment 2 of the North American isolate of ISAV. A $10 \mu$ aliquot of the reverse transcriptase reaction mixture was added to the PCR Master Mix containing $20 \mathrm{mM}$ Tris- $\mathrm{NaCl}, 50 \mathrm{mM} \mathrm{KCl}$, $1.5 \mathrm{mM} \mathrm{MgCl}_{2}$ and $0.2 \mathrm{mM}$ each of dNTPs. Primers were then added to a final concentration of $50 \mathrm{pmol}$. After addition of 2 drops of mineral oil, $0.5 \mu$ of AmpliTaq DNA Polymerase (Perkin-Elmer, Norwalk, CT, USA) was added with gentle mixing and the samples placed in a programmable thermal cycler (MJ Research). After an initial incubation at $95^{\circ} \mathrm{C}$ for $4 \mathrm{~min}$, the samples were subjected to 35 cycles of PCR $\left(94^{\circ} \mathrm{C}\right.$ for $45 \mathrm{~s}, 59^{\circ} \mathrm{C}$ for $45 \mathrm{~s}$, and $72^{\circ} \mathrm{C}$ for 1 min $45 \mathrm{~s}$ ) followed by incubation at $72^{\circ} \mathrm{C}$ for $7 \mathrm{~min}$. The samples then were electrophoresed in a Sea Plaque agarose gel containing ethidium bromide and examined under UV light. PCR amplification products were cut from the gel and processed for DNA sequencing

Sequencing: The nucleotide sequences of the RTPCR products were determined by the dye-terminator dideoxy system using an Applied Biosystems 373A automated DNA sequencer. A 1151 bp sequence was determined for the polymerase protein gene PB1 and a $436 \mathrm{bp}$ sequence was determined for the NS gene of the North American ISAV isolate, Nucleotide and deduced amino acid sequences of the North American
ISAV isolate were aligned and compared to the corresponding sequence previously published for the Norwegian strain using the Lasergene DNASTAR software package.

Results. RT-PCR was used to amplify a 1209 bp fragment from genome segment 2 and a 493 bp fragment from genome segment 8 of the North American isolate of ISAV. Genome segment 2 encodes a protein (PB1) and genome segment 8 encodes the NS protein(s) of orthomyxoviruses. Agarose gels of these RT-PCR amplification products are shown in Fig. 1. Sequences were determined for $1151 \mathrm{bp}$ of the cDNA from the polymerase gene and 434 bp of the NS gene.

Comparison of these partial cDNA nucleotide sequences (Figs. $2 \& 3$ ) with published sequences of ISAV from Norway confirmed that the North American virus isolate is ISAV. No areas of extensive sequence variation were observed between the 2 viruses. Rather, variations appeared as differences in 1 or 2 nucleotides and were more or less evenly distributed throughout the entire genomic region sequenced. The North American ISAV isolate exhibited $82.9 \%$ identity with the Sotra $92 / 93$ ISAV isolate from Norway in the polymerase protein PB1 gene. The 2 previously reported cDNA sequences of the NS gene of the same Norwegian ISAV isolate (Glesvaer/2/90) had an identity of $98.5 \%$ in the region examined. The major difference in

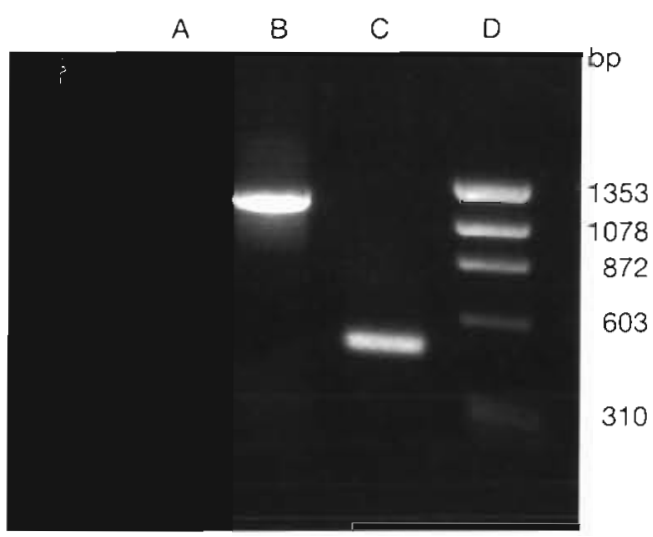

Fig. 1 Agarose gel of RT-PCR amplified cDNAs of North American isolate of ISAV. Lane A: negative control, unınfected CHSE cells; Lane B: polymerase PB1 gene; Lane C: NS gene; Lane D: molecular size markers 


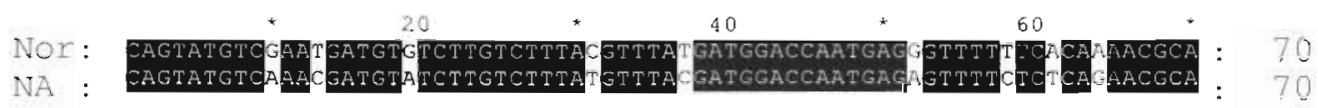

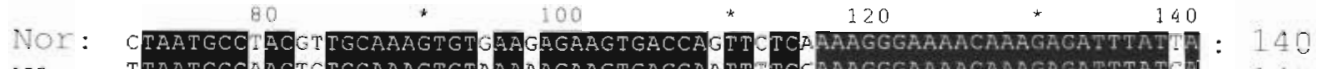

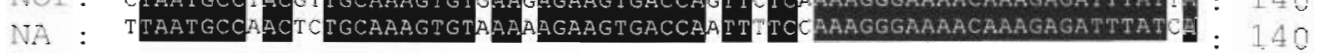

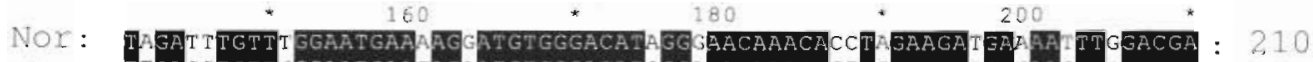

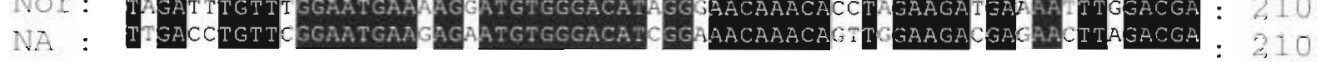

Nor: AACTGTTSGT STT GCAGACT TASGCETT GTGAAATACETGATAAACAACAAGTACGATGAGGCAGAGAAA : 280

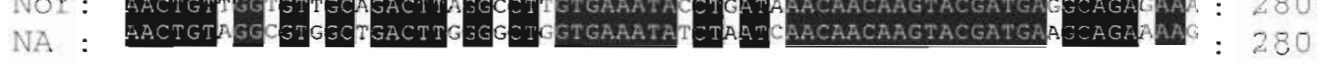

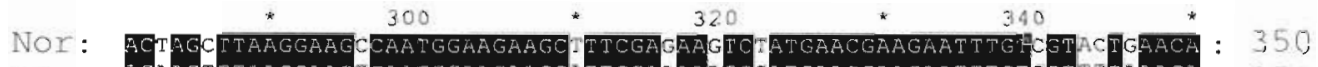

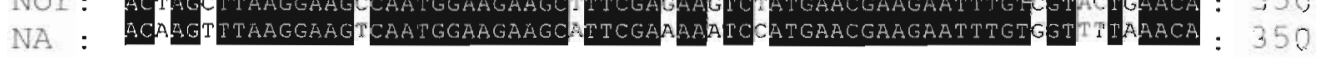

NNA: :

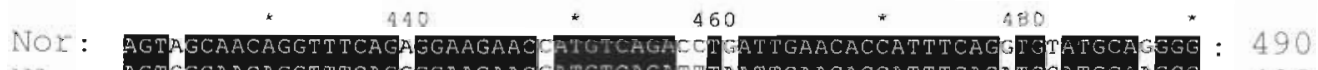

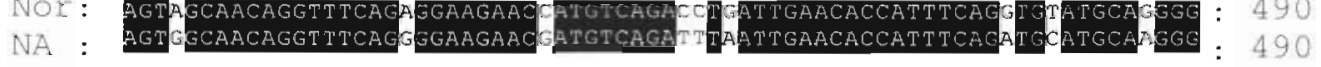

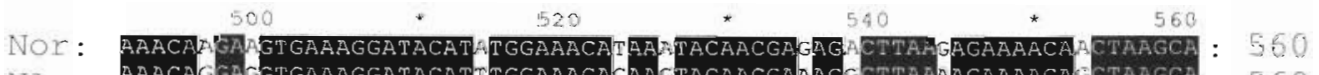

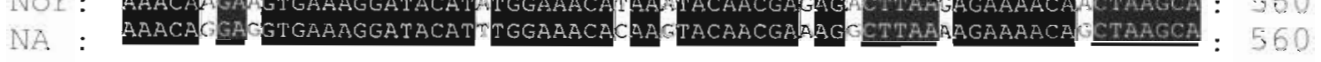

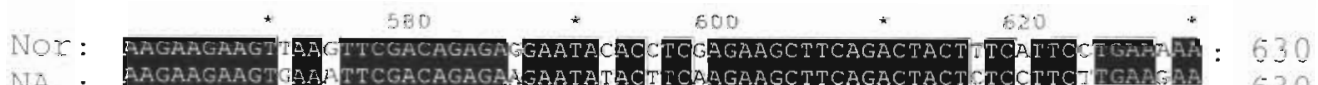

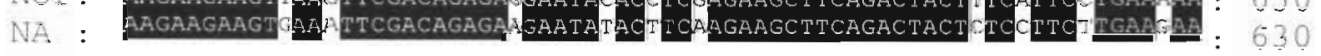

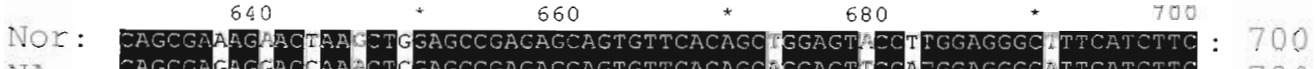

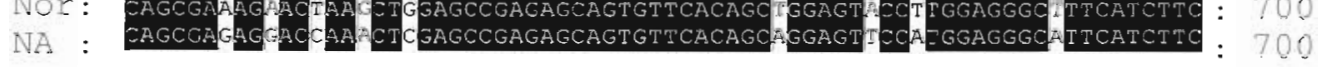

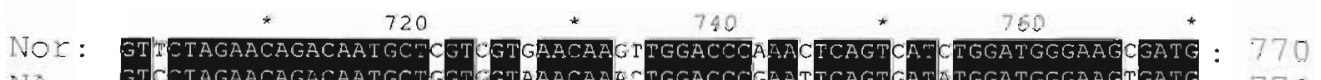

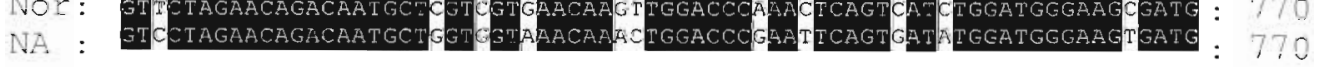

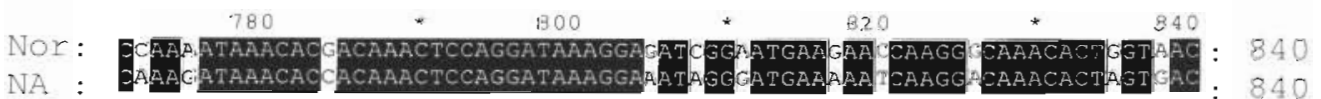

Fig. 2. Comparison of partial cDNA sequence of polymerase PB1 gene of North American isolate of ISAV (NA) with Norwegian ISAV strain Sotra 92/93 (Nor: sequence from Krossoey et al. unpubl., GenBank accession no. AJ002475)
Nor: GCTCACT NA : ACTCACA GGAGATACTCCAARTACAACGAGAGCATGTGCCCAGAGGTGATGATGCTG TCCTAAGAGAA : 910

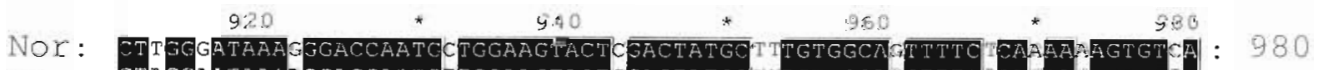

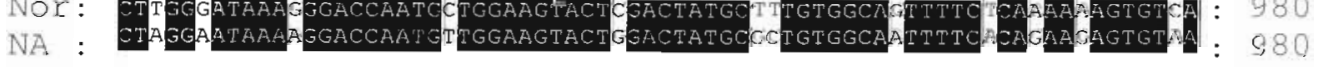

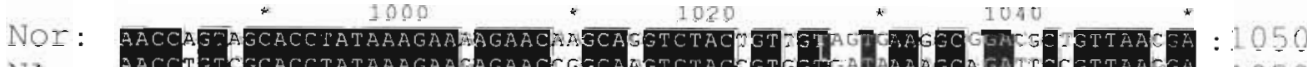

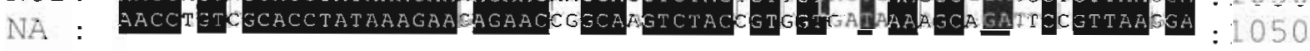

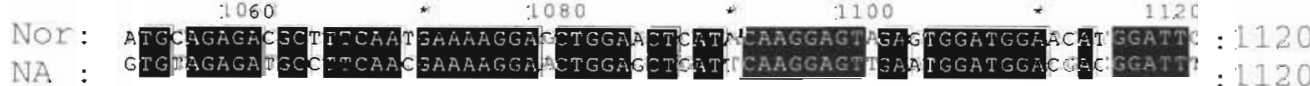

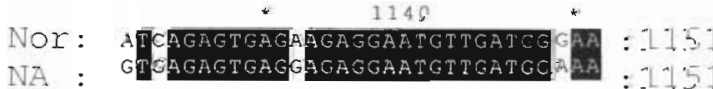




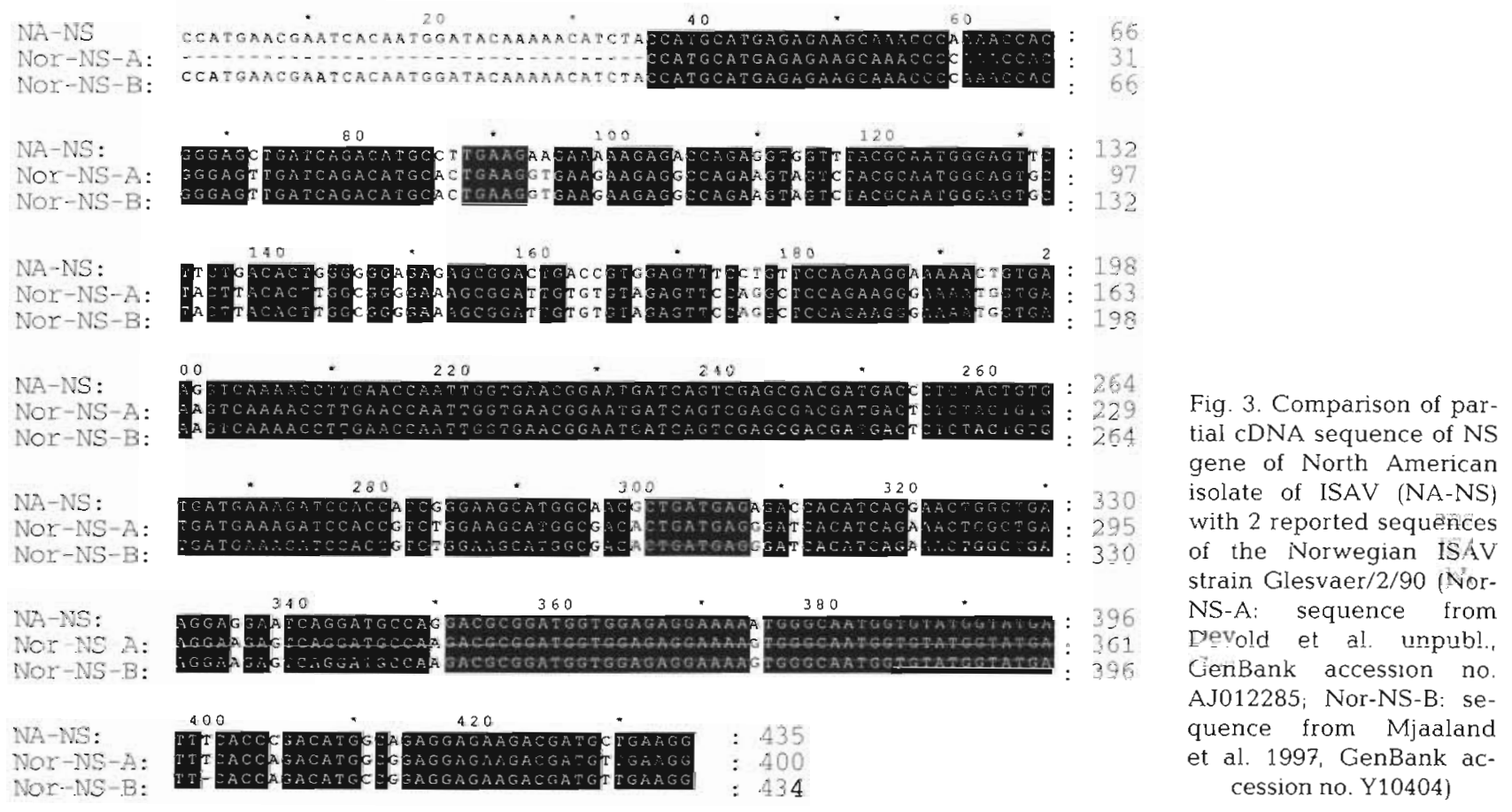

these 2 sequences of the same Norwegian isolate was the deletion of a single thymine at position 399 (Fig. 3) in the sequence reported by Mjaaland et al. (1997). Comparison of the CDNA sequence of the NS gene of the North American ISAV isolate with the Norwegian isolate NS gene revealed $88 \%$ identity with the sequence reported by Krossoey et al. (unpubl., GenBank accession no. AJ002475) and $89 \%$ identity with the sequence reported by Mjaaland et al. (1997).

The deduced amino acid sequences of the partial cDNA fragments of the North American and Nor- wegian ISAV isolates also were compared. The North American ISAV exhibited $96.6 \%$ similarity with the Sotra 92/93 ISAV isolate from Norway in the deduced amino acid sequences of the PB1 protein encoded by the partial cDNA of genome segment 2 (Fig. 4). The cDNA sequence of genome segment 8 contains 2 putative open reading frames (ORF) (Mjaaland et al. 1997 , GenBank accession no. Y10404; Devold et al. unpubl., GenBank accession no. AJ012285). The North American ISAV isolate exhibited only $71.2 \%$ (GenBank accession no. AJ012285) and 66.7\% (GenBank acces-

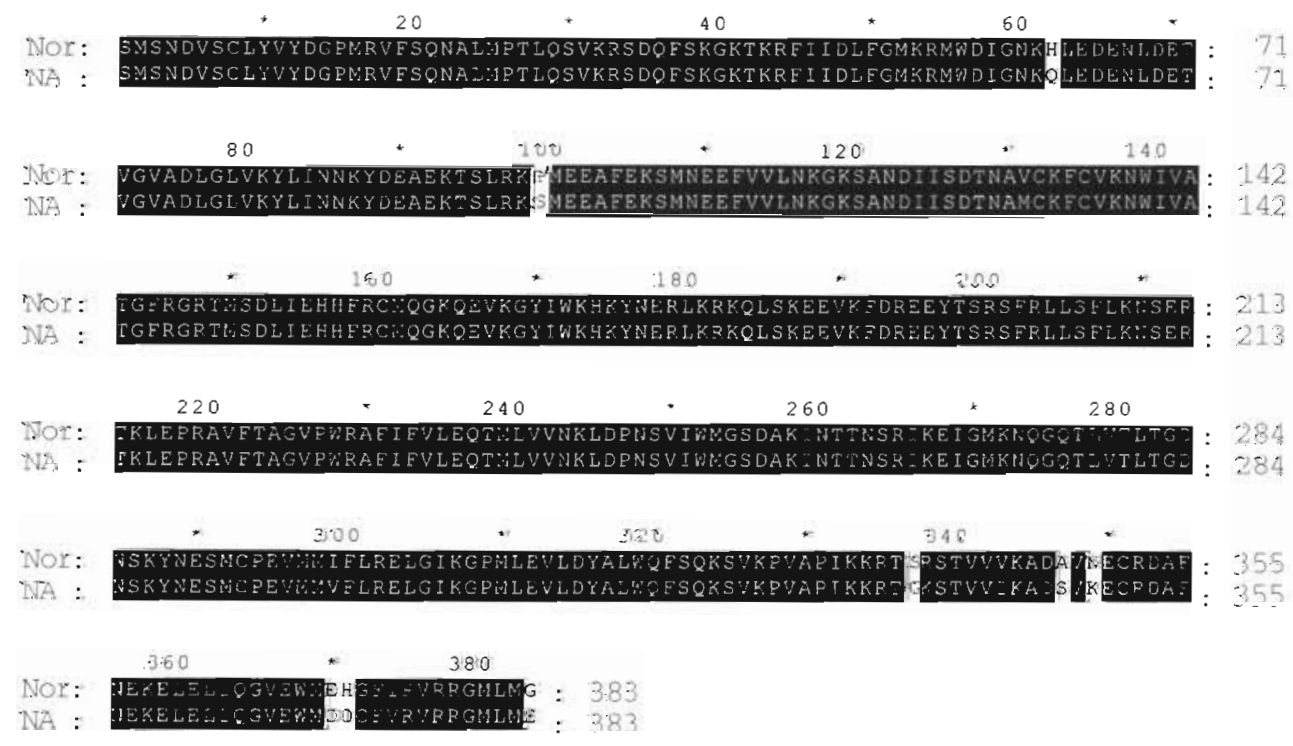

Fig. 4. Comparison of partial deduced amino acid sequence of PB1 protein of North American isolate of ISAV (NA) with Norwegian ISAV strain Sotra 92/93 (Nor: sequence from Krossoey et al. unpubl., GenBank accession no. AJ002475) 
Fig. 5. Comparison of partial deduced amino acid sequence of NS protein encoded in putative ORF 1 of North American isolate of ISAV (NA-NS-P1) with 2 reported sequences of the Norwegian ISAV strain Glesvaer/2/90 (Nor-NS-AP1 sequence from Devold et al. unpubl., GenBank accession no. AJ012285; Nor-NS$\mathrm{BP} 1$ : sequence from Mjaaland et al. 1997, GenBank accession no. Y10404)

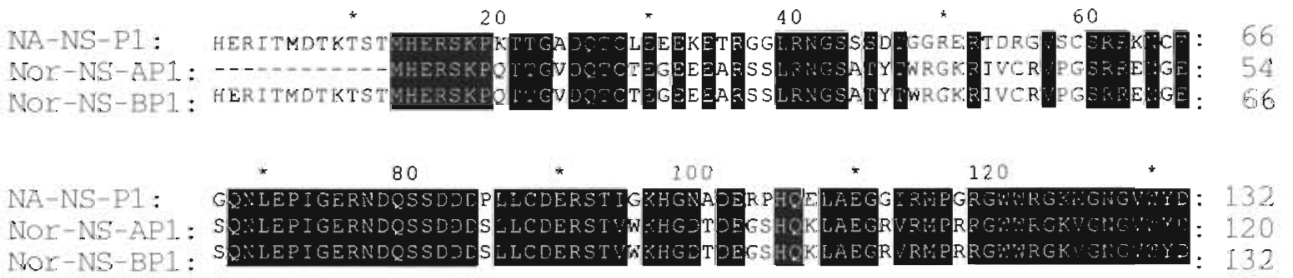

140

NA-NS-P1: FTRHGRGEDDAE : 144 Nor-NS-AP1: ETRHGGGEDDVE : 132 NOT-NS-BP1: SPDMPEEKTMLK : 144
Fig. 6. Comparison of partial deduced amino acid sequence of NS protein encoded in putative ORF 2 of North American isolate of ISAV (NA-NS-P2) with 2 reported sequences of the Norwegian ISAV strain Glesvaer/2/90 (Nor-NS-AP2: sequence from Devold et al. unpubl., GenBank accession no. AJ012285; Nor-NS-BP2: sequence from Mjaaland et al. 1997. GenBank accession no. Y 10404)
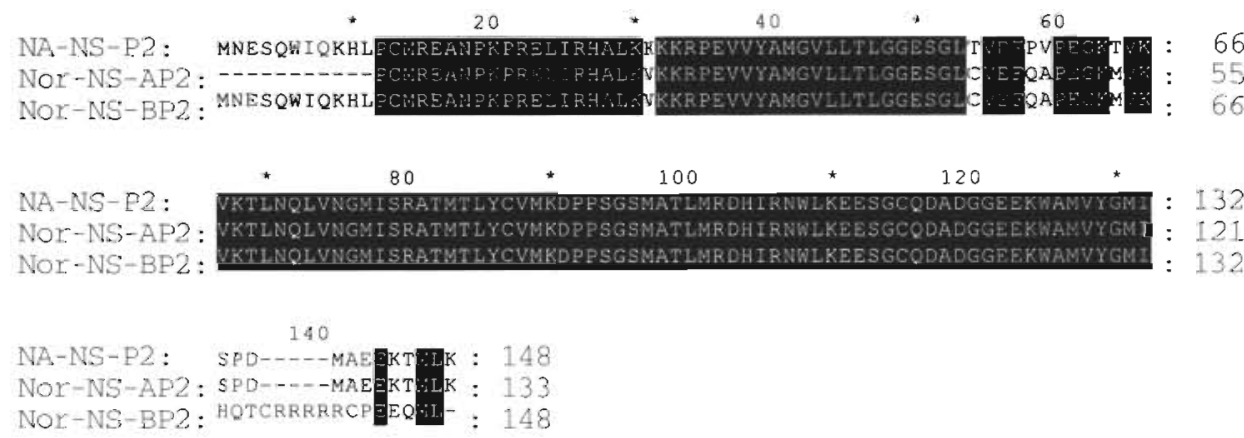

sion no. Y10404) similarity with the Norwegian isolate in the deduced amino acid sequence of the protein encoded in of the partial cDNA fragment of ORF 1 of genome segment 8 (Fig. 5). However, the North American ISAV isolate exhibited $96.2 \%$ similarity in the deduced amino acid sequences of the protein encoded in the partial cDNA of ORF 2 reported by Devold et al. (unpubl., GenBank accession no. AJ012285) and 87.2\% similarity with the sequence reported by Mjaaland et al. (1997, GenBank accession no. Y10404) (Fig. 6).

Discussion. The results of this investigation confirm that the virus isolated from cultured Atlantic salmon in the Bay of Fundy, New Brunswick, Canada, which exhibit a new disease syndrome, is similar to ISAV, an orthomyxo-like virus previously reported only in Norway. The North American ISAV isolate exhibited $82.9 \%$ identity with the Sotra $92 / 93$ ISAV isolate from Norway in the polymerase protein PB1 gene encoded in genome segment 2. Similarly, the North American ISAV isolate exhibited 88 and $89 \%$ identity in the partial nucleotide sequence of the NS gene (segment 8 ) with the 2 published sequences of the Glesvaer/2/90 ISAV isolate from Norway. The major difference between the 2 reported partial sequences of the NS gene of the same Norwegian isolate was the deletion of a single thymine at position 399 (Fig. 3) in the sequence reported by Mjaaland et al. (1997). The absence of this thymine deletion in the sequence of the North American ISAV isolate reported here suggests that this deletion is likely an error in the sequence reported by Mjaaland et al. (1997).

The deduced amino acid sequences of the partial PB1 protein of the North American ISAV and the Sotra $92 / 93$ Norwegian isolate were $96.6 \%$ similar. In addition, there was a $96.2 \%$ similarity between the partial NS protein encoded in ORF 2 of the North American ISAV and the sequence reported for the Norwegian Glesvaer/2/90 isolate by Devold et al. (unpubl., GenBank accession no. AJ012285). There was significantly lower similarity $(71.2 \%)$ between the deduced amino acid sequences of the partial NS protein encoded in ORF 1 of genome segment 8 of the North American ISAV and the Norwegian ISAV described by Devold et al. (unpubl., GenBank accession no. AJ012285).

Comparison of the deduced amino acid sequences of the PB1 protein of both ISAV isolates with PB1 of a variety of other orthomyxoviruses (data not shown) failed to reveal any regions of significant similarity and revealed an overall similarity of only approximately $16 \%$ with other orthomyxoviruses. No similarity was found between the NS proteins of ISAV and NS proteins of other orthomyxoviruses. Therefore, ISAV apparently is not closely related to other orthomyxoviruses in genomic sequences. More extensive studies 
of the genomic sequences of ISAV are required to permit a definitive determination of the phylogenetic relationships (e.g phylogenetic tree) between ISAV and other orthomyxoviruses.

Based on virion morphology and the number and size of genome segments, ISAV appears to resemble members of the Orthomyxoviridae. The nucleotide and deduced amino acid sequence comparisons of partial cDNA RT-PCR amplification products from 2 of the 8 genome segments of the North American virus isolate with the published sequences of the Norwegian isolates of ISAV confirm that the North American virus clearly is ISAV. However, the genomic sequence differences between the North American and Norwegian ISAV isolates revealed in this investigation suggest the possibility that the North American ISAV isolate may represent a distinct genomic variant, and the sudden appearance of ISAV in North America may not be the result of a recent importation of infected fish from Norway or a recent introduction of a Norwegian strain by naturally infected wild populations. However, little is known about possible reservoirs of ISAV in natural fish populations in Norway or in North America. Furthermore, these are the only isolates of ISAV for which genomic sequence information is available. Sequences of these and other genome segments should be determined for a variety of ISAV isolates both in Norway and in North America to determine the level of genomic variation among ISAV isolates in a given geographic area.

Nucleotide sequence accession number. The GenBank accession no. for the partial nucleotide sequence of the polymerase gene PB1 of the North American

Editorial responsibility: Jo-Ann Leong,

Corvallis, Oregon, USA isolate of ISAV described in this study is AF095254. The GenBank accession no. for the partial nucleotide sequence of the NS gene of the North American isolate of ISAV described in this study is AF095255.

Acknowledgements. This study was supported in part by the Maine Agricultural and Forestry Experiment Station (MAFES). MAFES publication 2298.

\section{LITERATURE CITED}

Blake S, Schill WB, McAllister P, Lee MK, Singer J, Nicholson BL (1995) Detection and identification of aquatic birnaviruses by polymerase chain reaction (PCR). J Clin Microb 33:835-839

Bouchard D, Keleher W, Opitz HM, Blake S, Edwards KC, Nicholson BL (1999) Isolation of infectious salmon anemia virus (ISAV) from Atlantic salmon in New Brunswick, Canada. Dis Aquat Org 35:131-137

Dannevig BH, Falk K, Namork E (1995) Isolation of the causal virus of infectious salmon anemia (ISA) in a long-term cell line from Atlantic salmon head kidney. J Gen Virol 76 : $1353-1359$

Hovland TA, Nylund K, Watanabe K, Endresen C (1994) Observation of infectious salmon anemia virus in Atlantic salmon, Salmo salar L. J Fish Dis 17:291-296

Lannan CN, Winton JR, Fryer JL (1984) Fish cell lines: establishment and characterization of nine cell lines from salmonids. In Vitro 20:107-114

Mjaaland S, Rimstad K, Falk K, Dannevig BH (1997) Genomic characterization of the virus causing infectious salmon anemia in Atlantic salmon (Salmo salar L.): an orthomyxolike virus in a teleost. J Virol 71:7681-7686

Nylund A, Hovland T, Watanabe K, Endresen C (1995) Presence of infectious salmon anemia virus (ISAV) in tissues of Atlantic salmon, Salmo salar L., collected during three separate outbreaks of the disease. J Fish Dis 18:135-145

Submitted: July 21, 1998; Accepted: December 2, 1998 Proofs received from author(s): January 21, 1999 


\section{Erratum}

\section{Isolation of infectious salmon anemia virus (ISAV) from Atlantic salmon} in New Brunswick, Canada

D. Bouchard, W. Keleher, H. M. Opitz, S. Blake, K. C. Edwards, B. L. Nicholson

Diseases of Aquatic Organisms 35:131-137, 1999

\section{Genomic relationships of the North American isolate of infectious salmon anemia virus (ISAV) to the Norwegian strain of ISAV}

S. Blake, D. Bouchard, W. Keleher, M. Opitz, B. L. Nicholson

Diseases of Aquatic Organisms 35:139-144, 1999

In both articles, an incorrect primer sequence appeared. The error does not change any results or conclusions.

- Page 132, under 'Materials and Methods; RT-PCR', lines $6-7$, the sentence should begin: 'The downstream primer (5'TAGGGGCATACATCTGCATC3') was designed...'.

That is, the correct primer sequence is TAGGGGCATA-

CATCTGCATC, and not the sequence published.
- Page 140 , in Table 1 , the downstream primer for NS gene should likewise be 5'TAGGGGCATACATCTGCATC3' and not the sequence published. 